\begin{tabular}{|c|c|c|c|}
\hline Eiszeitalter $u$. Gegenwart & $\mathbf{4 6}$ & $132-143$ & Hannover 1996 \\
\hline
\end{tabular}

\title{
Zur Problematik der pleistozänen und holozänen Vergletscherung Süd-Kamtschatkas - erste Ergebnisse bodengeographischer Untersuchungen
}

\author{
Wolfgang Zech, Rupert Bäumler, Oksana Savoskul \& Gerlinde Sauer*)
}

Pleistocene, Early Wisconsin, Holocene, Glaciation, Soil development, Kamchatka

\begin{abstract}
Kurzfassung: Im Plotnikovagebiet westlich von Petropavlovsk, Süd-Kamtschatka, lassen sich mindestens zwei Talmoränenkomplexe (M1 und M2) mit dazugehörigen Terrassensystemen nachweisen. Die älteren sogenannten M1-Moränen reichen bis auf ca. $300 \mathrm{~m}$ ü. M. herab; sie haben weiche, verwaschene Konturen. Die jüngeren M2Moränen schließen sich ab etwa 350 - $450 \mathrm{~m}$ an; ihr Relief ist unruhig, reich an Toteislöchern und Wallformen. Die Böden dieser Moränen und der korrespondierenden T1bzw. T2-Terrassen unterscheiden sich bezüglich Verwitterung und Verbraunung nur geringfügig. So weisen die M1bzw. T1-Böden kräftigere Kryoturbationen auf sowic deutlichere Verwitterungsrinden am Bodenskelett. Jedoch fichlen Hinweise auf eine interglaziale Überprägung z. B. in Form tonreicher Unterbodenhorizonte. Alle Böden weisen außerdem 3-4 Tephralagen auf, die auf folgende Eruptionen zurückgeführt werden können: Tephra 1 = Opala, 1400-1500 a BP; Tephra 2 = Ksudach 1, 1700-1800 a BP; Tephra 3 = Ksudach 2 = 6000 a BP; Tephra $4=$ Kuril Lake Il'inskay, 7600-7700 a BP (BrarTseva et al. 1992). Wir nehmen deshalb an, daß die M1-Moränen nicht ins Mittelpleistozän, sondern ins Spätpleistozän einzuordnen sind, und kein Interglazial sie von den M2-Moränen trennt. Vielmehr lassen die Befunde vermuten, daß die M1-Moränen während einer frühen Phase des Spätpleistozäns, die M2-Moränen während einer späteren Periode des Spätpleistozäns abgelagert wurden. Dieses Ergebnis macht wahrscheinlich, daß in Kamtschatka, im Gegensatz zu Mitteleuropa, die Gletscher in einer früheren Phase des Spätpleistozäns (vergleichbar dem Frühwürm) weiter ins Tal vorgestoßen sind,
\end{abstract} als während der später folgenden Perioden.

Tephrachronologische Untersuchungen einer Bodensequenz auf Stirnmoränen von 350-1000 m ü. M. im Topolovajatal ergeben, daß diese bis ca. $930 \mathrm{~m}$ ü. M. stets Tephra 1, 2, 3 und 4 aufweisen und damit älter als 7600/7700 Jahre BP sind, also zu hoch- und spätglazialen, evtl. auch frühholozänen Gletschervorstößen gehören. Erst die Stirnmoränen in ca. $980 \mathrm{~m}$ zeigen lediglich Tephra 1 und 2; sie sind somit älter als 1700/1800 Jahre BP, aber jünger als 6000 Jahre BP. Vermutlich korrelieren sie mit mittelholozänen Eisvorstößen. In ca. $1000 \mathrm{~m}$ Höhe liegen zwei weitere frische *) Anschriften der Verfasser: Prof. Dr. W. ZeCH, Dr. R. BÄUmler, G. SAuER, Lehrstuhl Bodenkunde und Bodengeographie, Universität Bayreuth, Postfach 101251, D-95440 Bayreuth. Dr. O. SAvoskul, Geographisches Institut, Russische Akademie der Wissenschaften, Staromonetny per. 29, Moskau, 109017
Wallmoränen mit initialer Bodenbildung, die nach den lichenometrischen Befunden während der sog. „kleinen Eiszeit" gebildet wurden. Tephralagen treten nicht mehr auf.

\section{[Pleistocene and Holocene glaciation in South} Kamchatka - first results of soil geographic studies]

Abstract: In the Plotnikova Valley, west of Petropavlovsk in South Kamchatka, two valley drift complexes can be identified. They are correlated with glaciofluvial terraces. The older, so called M1-moraines descend to about $300 \mathrm{~m}$ a.s.l. corresponding with the T1-terraces; they are smoothand vague-shaped, without kettles. The younger, so called M2-moraines descend to 350-450 $\mathrm{m}$ a.s.l.; their relief is well formed and rich in kettles and ridges, and they are connected with the T2-terraces. The soils developed on these drifts and terraces do not differ significantly with respect to colour and weathering intensity. Soils of the older drift and terraces reveal stronger cryogenetic disturbances and their boulders and gravels are characterized by more pronounced weathering crusts in comparison to the younger drift and terraces. No features indicating interglacial weathering can be identified in the subsoils. But all soils are stratified by 3-4 tephra layers, due to the following eruptions: tephra 1 = Opala, 1400-1500 a BP; tephra 2 = Ksudach 1, 17001800 a BP; tephra $3=$ Ksudach $2=6000$ a BP; tephra $4=$ Kuril Lake Il'inskay, 7600-7700 a BP (BraITSEva et al. 1992). According to these results it is supposed that both moraines indicate Late Pleistocene glaciation; the M1 drift was deposited during an early phase of the Late Pleistocene, the M2 drift characterizes a second phase of the Late Pleistocene, separated only by an interstadial, not by an interglacial. In contrast to Middle Europe, early Late Pleistocene glaciation has been more pronounced than Late Pleistocene second phase glaciation. Studying soil development on frontal moraines along a sequence from 350 to $1000 \mathrm{~m}$ a.s.l. in the Topolovaya-Valley it was found that up to about $930 \mathrm{~m}$ a.s.1. all soils are stratified by tephra 1-4 layers, indicating that the age of the corresponding moraines is older than $7600 / 7700$ a BP. Drift in about $980 \mathrm{~m}$ a.s.l. shows only tephra 1 and 2 . These moraines are older than 1700/1800 a BP, but younger than 6000 a BP. Probably they indicate a glacial advance during the middle Holocene (Neoglacial). In $1000 \mathrm{~m}$ a.s.l. two additional ridges can be identified, well formed with initial soil formation but without tephra. According to the lichenometric results they characterize snow and ice accumulations during the so-called "Little Ice Age". 


\section{Einleitung und Problemstellung}

Die Gebirge der Halbinsel Kamtschatka (geographische Breite etwa $51-61^{\circ} \mathrm{N}$, und geographische Länge um $160^{\circ} \mathrm{E}$ ) weisen verbreitet rezente Gletscher auf. Im Südteil reichen diese z. B. am Awatscha (2741 m ü. M.) in Form schuttbedeckter Loben bis auf ca. $800 \mathrm{~m}$ herab; am Klutschesvkaja (4750 m ü. M.) in Zentralkamtschatka finden sich perenne Schneeund Eisrinnen bis in Tiefen von ca. $1600 \mathrm{~m}$. Im Pleistozän waren auch die Tieflagen vergletschert, wie zahlreiche morphologische Befunde belegen. Dazu zählen trogförmige Talquerschnitte, Rundhöcker, Kare,
Transfluenz-Pässe, Toteislöcher und Moränen in Verbindung mit fluvioglazialen Schotterfluren. Dies wurde bereits früh erkannt und beschrieben (z. B. TscherbaKov 1938, 1940, 1941; Kuschev \& LiverovsKIJ 1938, 1940; Vlasov 1959a, 1959b; KOSCHEMIANKO 1966). Auch pollenanalytische Befunde belegen Kaltzeiten (Braitseva et al. 1968). Allerdings besteht keine Übereinstimmung bezüglich der Frage, wann und wie oft Kamtschatka während des Pleistozäns vergletschert war. Die umfangreichsten Untersuchungen stammen von Braitseva et al. (1968). Danach war Kamtschatka während des Frühpleistozäns nicht vergletschert, wohl u. a. eine Folge der zu die-

Tab. 1: Mögliche stratigraphische Zuordnung der M1- und M2-Talmoränen.

Table 1: Potential stratigraphical classification of the M1 and M2 moraines.

\begin{tabular}{|c|c|c|}
\hline Hypothese & M1-Moränen & M2-Moränen \\
\hline \multirow[t]{2}{*}{ A) } & $\begin{array}{l}\text { Spätpleistozän, Phase } 1 \\
\text { (Frühwürm) }\end{array}$ & $\begin{array}{l}\text { Spätpleistozän, Phase } 2 \\
\text { (Hochwürm) }\end{array}$ \\
\hline & \multicolumn{2}{|c|}{$\begin{array}{l}\text { Die mittelpleistozäne Vergletscherung war schwächer als die } \\
\text { spätpleistozäne, die ganz Kamtschatka bedeckte; die M1-Talmoränen } \\
\text { dokumentieren somit Grundmoränen der besonders mächtigen } \\
\text { Vergletscherung während der Phase } 1 \text { des Spätpleistozäns, } \\
\text { die annähernd dem Frühwürm entsprechen soll. }\end{array}$} \\
\hline \multirow[t]{2}{*}{ B) } & $\begin{array}{l}\text { Spätpleistozän, Phase } 1 \\
\text { (Frühwürm) }\end{array}$ & $\begin{array}{l}\text { Spätpleistozän, Phase } 2 \\
\text { (Hochwürm) }\end{array}$ \\
\hline & \multicolumn{2}{|c|}{$\begin{array}{l}\text { Die mittelpleistozäne Vergletscherung bedeckte ganz Kamtschatka und } \\
\text { reichte z. T. bis in das Meer hinein. }\end{array}$} \\
\hline C) & Mittelpleistozän & Spätpleistozän \\
\hline \multirow[t]{2}{*}{ D) } & $\begin{array}{l}\text { Spätpleistozän, Stadium } 1 \\
\text { der Phase } 2\end{array}$ & $\begin{array}{l}\text { Spätpleistozän, Stadium } 2 \\
\text { der Phase } 2\end{array}$ \\
\hline & \multicolumn{2}{|c|}{$\begin{array}{l}\text { Die dem Frühwürm entsprechende Phase } 1 \text { des Spätpleistozäns war } \\
\text { schwächer ausgeprägt, als die dem Hochwürm entsprechende Phase } 2 . \\
\text { Jedoch weist Phase } 2 \text { mehrere Stadiale auf. }\end{array}$} \\
\hline \multirow[t]{2}{*}{ E) } & $\begin{array}{l}\text { Spätpleistozän, Phase } 2 \\
\text { (Hochwürm) }\end{array}$ & Spätglazial \\
\hline & \multicolumn{2}{|c|}{$\begin{array}{l}\text { Die dem Frühwürm entsprechende Phase des Spätpleistozäns war } \\
\text { schwächer ausgeprägt, als die dem Hochwürm entsprechende Phase } 2 . \\
\text { Die M2-Moränen kennzeichnen ausgeprägte spätglaziale Eisvorstöße, } \\
\text { deren Maximum fast die Ausdehnung der spätpleistozänen Vereisung } \\
\text { erreichte. }\end{array}$} \\
\hline \multirow[t]{2}{*}{ F) } & Spätglazial, Stadium 1 & Spätglazial, Stadium 2 \\
\hline & \multicolumn{2}{|c|}{$\begin{array}{l}\text { Die Vereisung während des maximalen Gletschervorstoßes im Spät- } \\
\text { pleistozän bedeckte ganz Kamtschatka; die hier beschriebenen Tal- } \\
\text { moränen kennzeichnen spätglaziale Eisvorstöße. }\end{array}$} \\
\hline
\end{tabular}




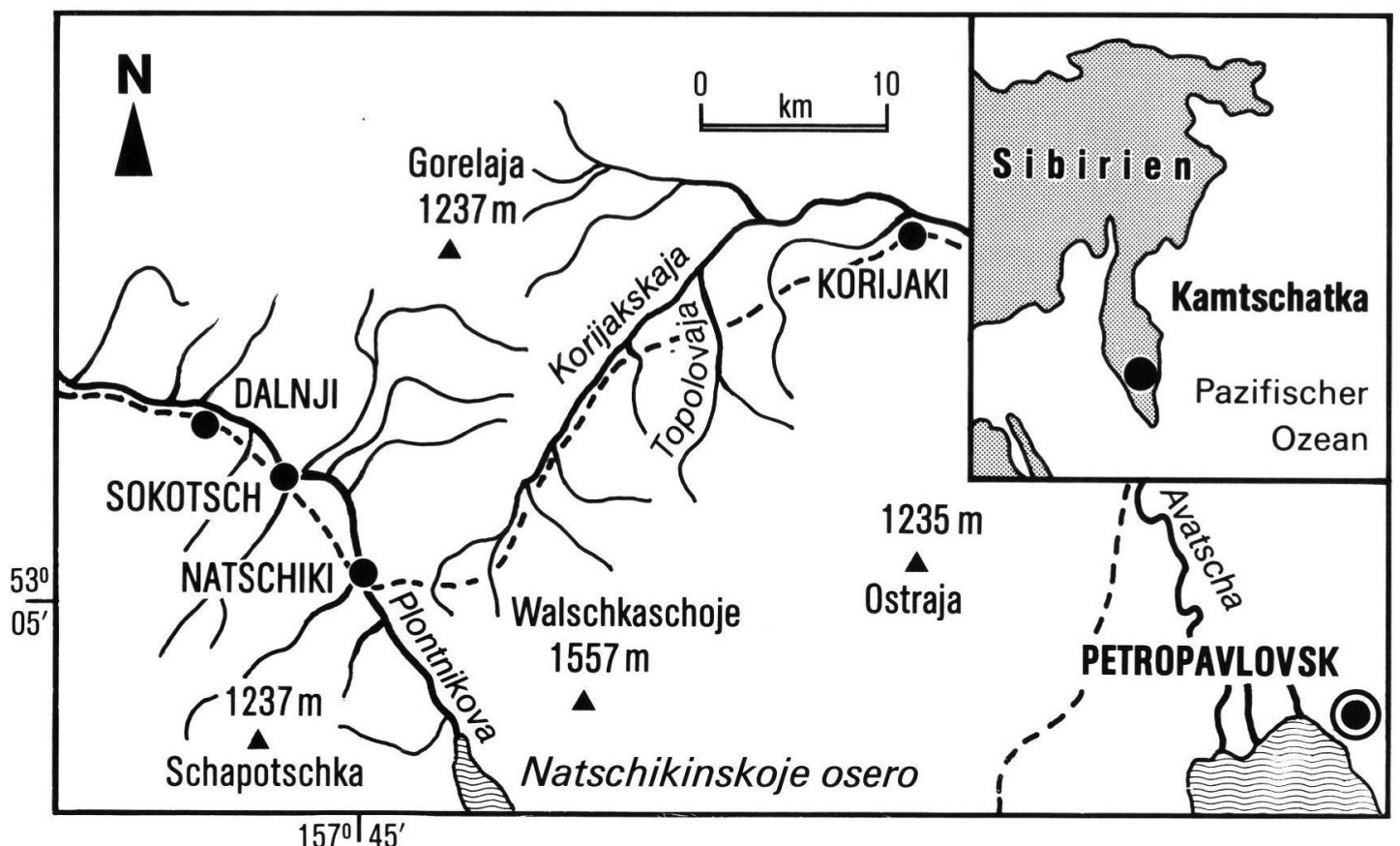

Abb. 1: Lage der Arbeitsgebiete im Plotnikova-Tal südlich Natschiki, und im Topolovaja-Tal. Kartenausschnitt aus Generalnji schtab Nr. 14-75-3/NS7-B, Petropavlovsk-Kamtschatskji, 1:500000, 1963.

Fig. 1: Location of the working area in the Plotnikova Valley (south of Natschiki) and the Topolovaja Valley.

ser Zeit noch geringen tektonischen Heraushebung. Im Gegensatz dazu gab es im Mittelpleistozän eine kräftige Vergletscherung, von der aber keine Moränen erhalten sein sollen, lediglich Zeugen in Form "alter" Trogtäler und fossiler, in der Kamtschatkadepression begrabener Sedimente. Während des Spätpleistozäns soll fast die gesamte Halbinsel (z. T. bis ins Meer hinein) von einem Eispanzer bedeckt gewesen sein. Die mittelpleistozänen Vergletscherungen hätten danach eine geringere Ausdehnung ausgewiesen als die spätpleistozänen.

Die in den Tallagen verbreitet vorkommenden, auf kräftige Talvergletscherung zurückzuführenden Moränen datieren Braitseva et al. (1968) ebenfalls ins Spätpleistozän. Der tiefer reichende Moränenkomplex (hier M1-Moränen genannt; bis auf ca. $3400 \mathrm{~m}$ ü. M.) ist morphologisch wenig differenziert, wirkt verwaschen und läßt keine Wallstrukturen erkennen. Die unmittelbar talaufwärts, d. h. wenig höher liegenden sogenannten M2-Moränen, sind morphologisch frisch und weisen deutliche, teilweise gestaffelte Wallstrukturen sowie Toteislöcher und Umfließungsrinnen auf. Nach Braitseva et al. (1968) gehören diese beiden Moränenkomplexe zu einer Kaltzeit. Im Gegensatz dazu bezeugen nach VLASOV \& Tschemenkov (1950), sowie Vlasov (1959 a, b) und LAPSCHIN (1963) die beiden Moränengenerationen zwei eigenständige Kaltzeiten, vermutlich mittelund spätpleistozänen Alters.

In Gesprächen mit Frau Dr. O. A. Braitseva, Herrn
Dr. I. V. Melekestsev (Institute of Volcanic Geology and Geochemistry, Petropavlovsk) und Herrn Dr. J. D. Muravjev (Institute of Volcanology, Petropavlovsk) wurden noch weitere Hypothesen diskutiert, die in Tabelle 1 zusammengefaßt sind.

Für das Holozän belegen Braitseva et al. (1968) zwei Gletschervorstöße. Den ersten um etwa 2000 Jahre BP, den zweiten um 1850. Solomina et al. (1995) konnten darüber hinaus aufgrund lichenometrischer Erkundungen einen dritten Vorstoß vor etwa 300 Jahren wahrscheinlich machen.

Ausgehend von diesen Befunden war es das Ziel unserer Untersuchungen, folgende Fragen zu überprüfen:

1. Wie unterscheiden sich die Böden der beiden Moränenkomplexe sowie jene der dazugehörigen T1- und T2-Terrassen vom Profilaufbau her, und lassen sich daraus Hinweise auf das relative Alter der entsprechenden Sedimente ableiten?

2 . Wie verändert sich der Profilaufbau der Böden mit zunehmender Höhenlage, und ergeben sich daraus Rückschlüsse auf eventuelle holozäne Gletschervorstöße?

\section{Ergebnisse}

\subsection{Bodenbildung auf M1- und M2-Moränen} sowie auf T1- und T2-Terrassen im Plotnikova-Tal

Als Arbeitsschwerpunkte wählten wir das Plotnikova- sowie das Topolovaja-Tal, ca. 90 bzw. $60 \mathrm{~km}$ 
$\mathrm{S}$

M2 - Seitenmoränen

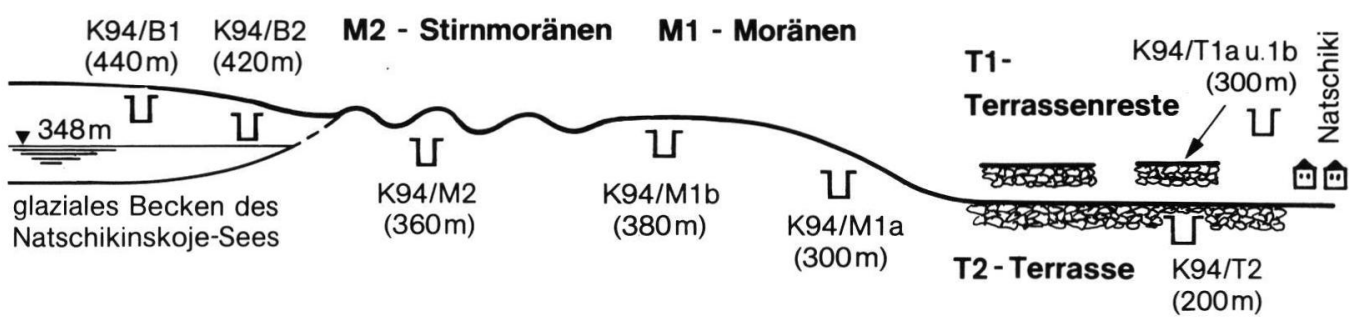

Abb. 2: Schematische Darstellung glazialer und fluvioglazialer Formen im Plotnikovatal, 90 km westlich von Petropavlovsk, Südkamtschatka (= Bodeneinschläge, B = Bohrungen).

Fig. 2: Schematic sketch of glacial and fluvioglacial deposits in the Plotnikova Valley, $90 \mathrm{~km}$ west of Petropavlovsk, South-Kamchatka ( 乙r = soil pits, $\mathrm{B}=$ auger profiles).

westlich von Petropavlovsk (Abb. 1). Betrachten wir zunächst das Plotnikova-Tal. Der Fluß Plotnikova entspringt im Zungensee-Becken des Natschikinskoje Sees. Das weite Tal der Plotnikova wird durch einen tektonischen Graben entlang einer nordwestlich verlaufenden Verwerfungslinie gebildet. Südlich der Ortschaft Natschiki weist das Plotnikova-Tal in etwa $200 \mathrm{~m}$ ü. M. ausgedehnte Schotterflächen auf, und zwar ca. $10 \mathrm{~m}$ über den rezenten Flußläufen (T2-Terrassen), die überwiegend landwirtschaftlich genutzt werden. Vereinzelt sind etwa $2 \mathrm{~km}$ nordwestlich des Ortes Sokotsch etwa $20 \mathrm{~m}$ über dem rezenten Flußniveau Reste älterer Terrassen nachweisbar (T1-Terrassen).

Etwa $3 \mathrm{~km}$ südlich von Natschiki gehen im oberen Plotnikova-Tal die T2-Schotter über in die sogenannte M1-Moräne, die kein ausgeprägtes Mikrorelief erkennen läßt, dagegen geglättet, abgeflacht und „verwaschen" wirkt. Es fehlen wallförmige Ablagerungen und Toteislöcher. Noch weiter südlich folgen talaufwärts in ca. $350-340 \mathrm{~m}$ ü. M. mehrere wallförmige Strukturen, die wir einem weiteren Moränenkomplex (M2) zuordnen und als Stirnmoränen interpretieren. Sie umspannen z. T. bogenförmig das glaziale Becken des Natschikinskoje-Sees, der vom Plotnikova-Fluß nach Nordwesten entwässert wird. Besonders auf der NW-Seite des Seebeckens gehen diese Stirnmoränen in typische Ufermoränen über. Abb. 2 zeigt eine schematische Skizze mit der Abfolge der verschiedenen glazialen und fluvioglazialen Formen des Plotnikova-Tals südlich Natschiki, sowie die Punkte, wo Bodenprofile angelegt, bzw. Bohrprofile beschrieben wurden. Die Höhe der Gipfelflur der umgebenden Berge liegt im Arbeitsgebiet zwischen 1000-1500 m ü. M. Rezente Vergletscherung ist nicht mehr vorhanden. Die Profile der T1-Terrasse wurden $2 \mathrm{~km}$ südwestlich von Sokotsch auf den Resten des $20 \mathrm{~m}$ Terrassenniveaus angelegt.
Profil T2 wurde direkt bei Natschiki aufgenommen. Die Profile M1a und M1b befinden sich südlich von Natschiki in Ober- und Mittelhanglage der M1-Ablagerungen, M2 wiederum südlich davon am Oberhang eines Stirnmoränenwalls des M2-Moränenkomplexes.

Bevor wir auf Einzelheiten des Profilaufbaus der Moränen und Terrassen im Plotnikova-Tal eingehen, erscheint es sinnvoll, die Tephra-Stratigraphie der Böden in Süd-Kamtschatka nach Braitseva et al. (1968, 1992) zu erklären. Wie in Abb. 3 dargestellt, finden sich in ungestörter Lagerung bis zu vier Leittephraablagerungen, deren ${ }^{14} \mathrm{C}$-Alter die Zeitspanne von $1400 / 1500$ bis $7600 / 7700$ Jahre BP umfaßt. Tephra 1, 2 und 4 sind i. d. R. hellgrau, während die besonders mächtige Fephra 3-Lage rötlich gefärbt und an der Basis grobkörniger ist. Tephra 4 ist nur schwach ausgeprägt, z. T. fehlt sie ganz.

Betrachten wir im folgenden zunächst die Horizontfolge der Böden auf der T2-Terrasse (Abb. 4, Tab. 2), so erkennt man eindeutig die Tephra 1-, Tephra 2und Tephra 3-Lagen, während Tephra 4 nicht mit Sicherheit auszumachen ist. Zwischen Tephra 1 und Tephra 2, sowie zwischen Tephra 2 und Tephra 3 haben sich fossile Ah-Horizonte erhalten, ebenso unterhalb Tephra 3. Der IV Ah-Horizont unterhalb Tephra 3 (Abb. 4) dokumentiert maßgeblich die Bodenbildung während des holozänen Klimaoptimums, während die III Ah- und III AB-Horizonte zwischen 6000-1700/1800 a BP gebildet wurden. Der geringmächtige II Ah-Horizont zwischen Tephra 1 und Tephra 2 hatte dagegen nur eine kurze Entwicklungszeit von ca. 300 Jahren. Unterhalb des IV Ah-Horizontes folgen rostfleckige, relativ geringmächtige verbraunte Lagen, mit vielfach hochkant gestellten Steinen und plattigem Gefüge sowie schwachen kryoturbaten Verwürgungen, bis dann in etwa $80-100 \mathrm{~cm}$ Bodentiefe die Schotter der T2Terrasse beginnen. Diese Befunde machen wahr- 


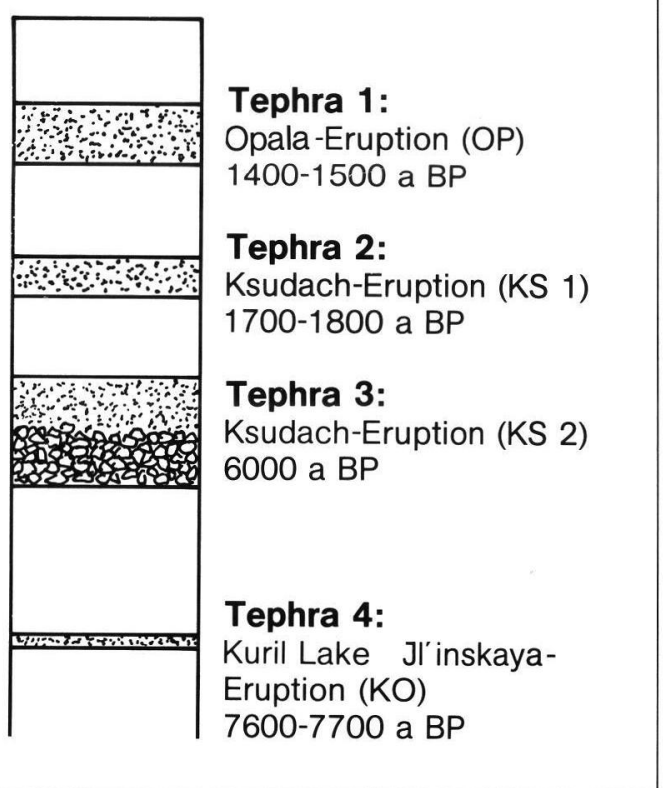

Abb. 3: Abfolge der Tephra-Lagen in ungestörten Bodenprofilen Süd-Kamtschatkas, ihr ${ }^{14} \mathrm{C}$-Alter sowie die zugehörigen vulkanischen Ereignisse (nach BralTSEva et al. 1968, 1992).

Fig. 3: Tephra-stratigraphy of undisturbed soil profiles in South-Kamchatka, their radiocarbon age, and corresponding volcanic events (according to Braltesva et al. 1968, 1992).

scheinlich, daß nach Ablagerung der T2-Schotter, während der Frühphasen der Bodenbildung intensiver Frostwechsel herrschte; bodengenetisch gesehen, entwickelten sich damals Tundrengleye. Die Existenz geringmächtiger fossiler Ah-Lagen im V GCv-Horizont weist auf kürzere, wärmere Zeitabschnitte hin.

Einen ähnlichen Profilaufbau weisen die Böden der T1-Terrasse auf, jedoch deuten sich im Vergleich zur T2-Terrasse folgende Unterschiede an (Abb. 4, Tab. 2):

- Die verbraunten Horizonte unterhalb Tephra 3 sind etwas mächtiger, jedoch nicht tonreicher. Die Verbraunung selbst ist nicht besonders intensiv. Die $\mathrm{Fe}_{\mathrm{O}} / \mathrm{Fe}_{\mathrm{d}}$-Quotienten der unterhalb Tephra 3 folgenden Horizonte schwanken in Profil K 94/T2 zwischen 0.23-0.58, in Profil K94/T1a zwischen 0.270.60 und in Profil K94/T1b zwischen 0,55-0,83. Auch die $\mathrm{Fe}_{\mathrm{d}}$-Werte (Abb. 5) lassen keine Hinweise auf besonders intensive Verwitterung der Unterböden von T1-Terrassen erkennen. Stets finden sich die höchsten $\mathrm{Fe}_{\mathrm{d}}$-Werte in den jüngeren T2-Böden. Die T1-Schotter beginnen zwischen 100-170 cm Bodentiefe, während auf der T2-Terrasse die Schotter zwischen $80-100 \mathrm{~cm}$ Tiefe anstehen.

- Die kryoturbaten Störungen der Unterbodenhorizonte der T1-Böden sind wesentlich ausgeprägter.
Auch größere Gesteinsbruchstücke sind dort senkrecht gestellt; $z$. T. weisen sie Frostsprengung auf. - Die T1-Schotter der Unterboden-Horizonte lassen z. T. Verwitterungsrinden erkennen, einzelne Steine sind mürbe, bzw. in situ verwittert, jedoch sind die entsprechenden Horizonte nicht stärker verlehmt und verbraunt.

Ergebnisse dieser Art machen wahrscheinlich, daß zwischen der Ablagerung der T1- und T2-Schotter kein längerer, wärmerer Zeitabschnitt in Form eines Interglazials lag. Nach ARKHIPOv et al. (1986) ist nämlich davon auszugehen, daß das Interglazial zwischen Mittel- und Spätpleistozän in NE-Sibirien wärmer war als die Jetztzeit, was sich in Form einer intensiveren Verbraunung und Verlehmung in den T1Unterböden nachweisen lassen sollte (BÄUMLER et al. 1991). Wohl aber sind unsere Befunde mit der Existenz eines Interstadials zwischen der Ablagerung der T1- bzw. T2-Terrassen gut zu erklären.

Wenden wir uns nun der Bodenbildung auf den Moränen zu. Das Profil K94/M1a in Hanglage (Abb. 4, Tab. 2) weist wie die Schotterböden die drei jüngeren Tephralagen 1, 2 und 3 auf, jedoch ist Tephra 2 nicht band- bzw. lagenförmig erhalten, sondern diskontinuierlich, vermutlich durch Solifluktion ge-

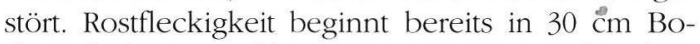
dentiefe (= III GBAh). Hangwasserzug tritt über dem stark verdichteten Unterboden auf. Unterhalb Tephra 3 folgt zunächst - allerdings nur stellenweise der IV Ah-Horizont, dessen Genese wir in das holozäne Klimaoptimum legen. Der nächst tiefere V GBv-Horizont markiert einen Schichtwechsel. Er ist etwas kräftiger braun gefärbt, wenig tonreicher und dichter als die hangenden Horizonte. Die obere Grenze zur IV Ah- bzw. zur Tephra 3-Lage bilden kantige, horizontal eingeregelte Gesteine. Die Aggregatoberflächen weisen Toncutane auf. Diese finden sich, wenn auch schwächer ausgebildet, im tieferen, noch verbraunten, skelettreichen und sehr dicht gelagerten V GCvBv-Horizont. Die Untergrenze der Verbraunung ließ sich wegen der zementartigen Verdichtung nicht eindeutig feststellen. Auffallend sind die vereinzelt vorkommenden Verwitterungsrinden der Steine, was mit den Befunden auf der T1-Terrasse übereinstimmt.

Derartige Verwitterungsrinden kennzeichnen auch das Bodenskelett der VI GCv- und IV G Cn-Horizonte von Profil K94/M1b (Abb. 4, Tab. 2). Im Gegensatz zum K94/M1a-Profil in Hanglage, weist das in schwach konkaver Geländeposition angelegte Profil K94/M1b im Unterboden (VI G Bv) nur eine relativ geringmächtige Verbraunung auf. Toncutane konnten wir nicht identifizieren.

Dies gilt auch für die Bodenprofile auf M2-Stirn- und Ufermoränen (Abb. 4, Tab. 2). Ihre Oberböden lassen stets die jungen Tephralagen 1, 2 und 3 erkennen. Darunter folgen zunächst humose Horizonte 


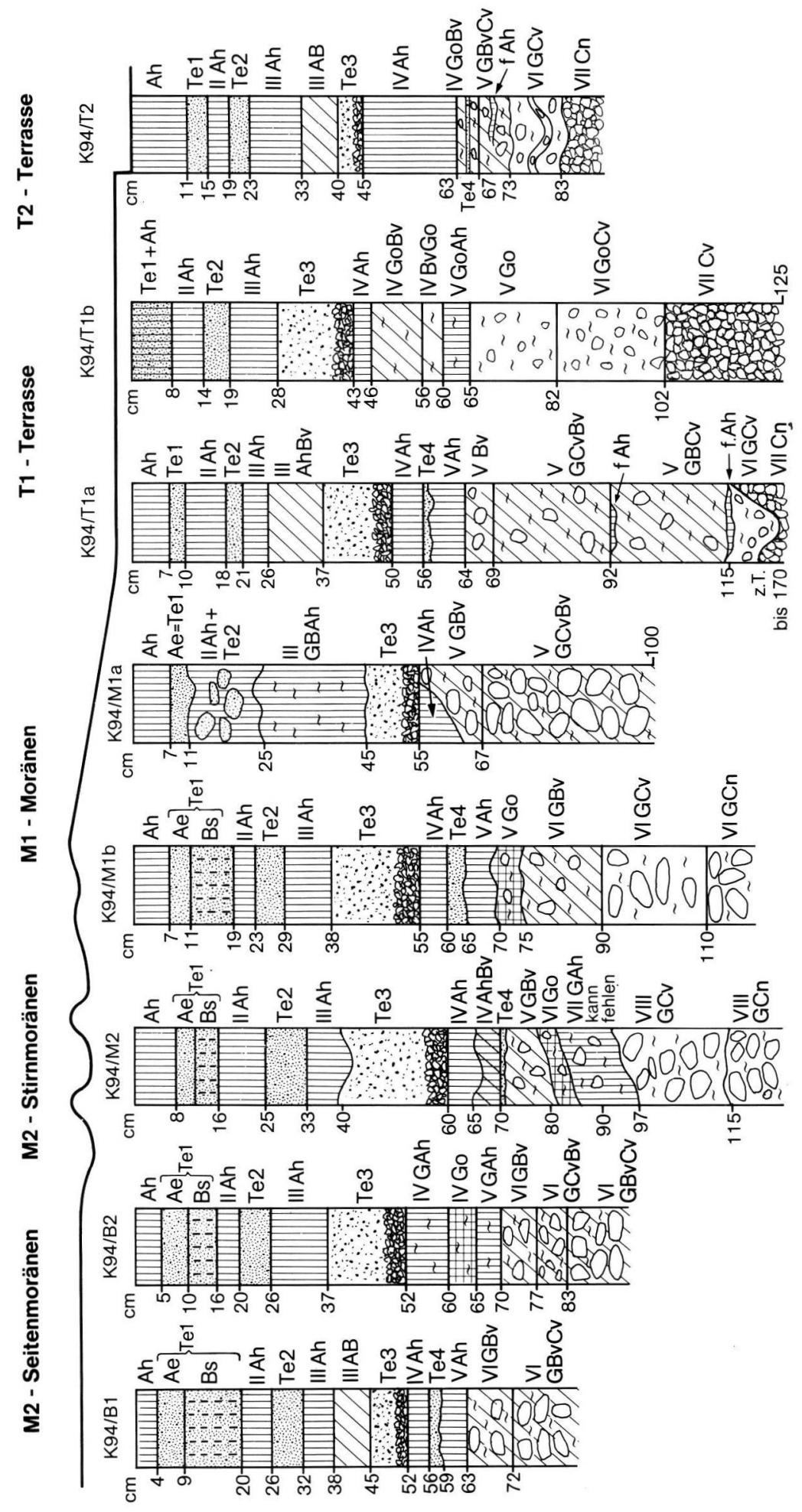




\begin{tabular}{|c|c|c|c|c|c|c|c|c|c|c|c|c|c|c|c|c|}
\hline $\begin{array}{l}\text { Profil Nr. } \\
\text { Morphol.Einheit }\end{array}$ & $\begin{array}{l}\text { Horizont- } \\
\text { folge }\end{array}$ & $\begin{array}{l}\text { Mächtigkeit } \\
\mathrm{cm}\end{array}$ & Farbe & Textur & Gefüge & $\begin{array}{l}\text { Skeleit } \\
\text { Vol\% }\end{array}$ & Dichte & besoricierheiten & $\begin{array}{l}\mathrm{pH} \\
\mathrm{CaCl}_{2}\end{array}$ & $\% \mathrm{C}$ & $\% \mathrm{~N}$ & $C / N$ & $\mathrm{Fe}_{\mathrm{d}}$ & $\mathrm{Fe}_{0}$ & $\frac{\mathrm{Fe}_{\mathrm{o}} \times \frac{1}{\mathrm{Fe}_{\mathrm{d}}}}{}$ & 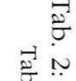 \\
\hline K 94/T2 & Ah & $0-11$ & $7.5 Y R 2 / 2$ & suL & krü & 0 & sl & & 4.12 & 11.39 & 0.95 & 12.0 & 7.0 & 4.3 & 61.4 & \\
\hline \multirow[t]{11}{*}{ T2-Terrasse } & Te1 & $11-15$ & $7.5 Y R 4 / 4$ & $S$ & $\sin$ & 0 & sl & & 4.41 & 1.70 & 0.15 & 11.3 & 3.7 & 2.8 & 75.7 & \\
\hline & $\|$ Ah & $15-19$ & 7.5YR $2 / 2$ & sul & subpol & 0 & 1 & & 4.39 & 5.85 & 0.45 & 13.0 & 8.1 & 6.4 & 79.0 & \\
\hline & Te2 & $19-23$ & $7.5 Y R 3 / 4$ & $S$ & $\sin$ & 0 & 1 & & 4.60 & 2.78 & 0.22 & 12.6 & 6.3 & 4.7 & 74.6 & \\
\hline & III Ah & $23-33$ & 7.5YR $2 / 2$ & $u$ & subpol & 0 & 1 & & 4.62 & 8.16 & 0.59 & 13.8 & 14.3 & 21.7 & 151.7 & 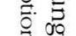 \\
\hline & III $A B$ & $33-40$ & $7.5 Y R 3 / 4$ & $\mathrm{sU}$ & subpol & 0 & 1 & & 4.80 & 6.80 & 0.42 & 16.2 & 20.2 & 16.2 & 80.2 & \\
\hline & Te3 & $40-45$ & $7.5 Y R 4 / 6$ & sU & krü & 0 & 1 & & 4.91 & 5.72 & 0.37 & 15.5 & 24.7 & 19.1 & 77.3 & \\
\hline & IV Ah & $45-63$ & $7.5 Y R 2 / 3$ & U & subpol & $<5$ & 1 & Skelettlängsachsen senkr. & 4.85 & 8.80 & 0.54 & 16.3 & 32.8 & 18.2 & 55.5 & 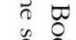 \\
\hline & IV GoBv & $63-67$ & $7.5 Y R 3 / 4$ & $u$ & subpol & $<5$ & 1 & $\cdot "$-, rostfleckig & 4.88 & 6.03 & 0.38 & 15.9 & 51.1 & 12.0 & 23.5 & $\stackrel{0}{0}$ \\
\hline & v GBvCr & $67-73$ & $2.5 Y R 4 / 4$ & $\mathrm{sU}$ & plat & 10 & 1 & dünne fAh-Hor., schw.rostfl. & 5.06 & 1.56 & 0.13 & 12.0 & 7.7 & 4.5 & 58.4 & 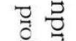 \\
\hline & VIGCV & $73-83$ & $7.5 Y R 4 / 4$ & us & plat & 40 & $\mathrm{md}$ & Kryoturbationen, schw.rostfl. & 4.94 & 0.27 & 0.03 & 9.0 & 7.5 & 3.2 & 42.7 & \\
\hline & VII Cn & $83-100+$ & & $s$ & $\sin$ & 80 & 1 & & & & & & & & & \\
\hline $\mathrm{K} 94 / \mathrm{T} 1 \mathrm{a}$ & Ah & $0-7$ & $7.5 Y R 3 / 2$ & fs & fkrü & 0 & sl & & & & & & & & & \\
\hline \multirow[t]{14}{*}{ T1-Terrasse } & Te1 & $7-10$ & $7.5 Y R 6 / 2$ & s & $\sin$ & 0 & sl & & & & & & & & & \\
\hline & $\|$ Ah & $10-18$ & $5 Y R 4 / 3$ & sul & subpol-krü & 0 & 1 & & & & & & & & & \\
\hline & Te2 & $18-21$ & 7.5 YR $5 / 6$ & suL & sin-subpol & 0 & 1 & & & & & & & & & \\
\hline & III Ah & $21-26$ & $2.5 Y R 4 / 2$ & U & krü-subpol & 0 & 1 & & & & & & & & & \\
\hline & III AhBv & $26-37$ & $7.5 Y R 4 / 4$ & $u$ & krü-subpol & 0 & 1 & & & & & & & & & \\
\hline & Te3 & $37-50$ & 5YR 4/8 & $\mathrm{sU}$ & fkrü & 0 & 1 & & & & & & & & & \\
\hline & IV Ah & $50-56$ & $7.5 Y R \quad 4 / 4$ & $s^{\prime} U$ & subpol & 0 & 1 & & 5.05 & 3.03 & 0.24 & 12.6 & 20.5 & 12.3 & 60.0 & \\
\hline & Te4 & $56-59$ & 10YR $5 / 6$ & $s^{\prime} U$ & subpol & 0 & 1 & & 5.04 & 2.72 & 0.19 & 14.3 & 14.2 & 10.4 & 73.2 & \\
\hline & VAh & $59-64$ & 1OYR $4 / 4$ & s'U & subpol & 0 & 1 & & 4.95 & 2.10 & 0.16 & 11.7 & 19,4 & 20,7 & $i 06.7$ & \\
\hline & VBv & $64-69$ & 10YR 5/4 & $s^{\prime} U$ & subpol & $<5$ & 1 & Skelettlängsachsen senkr. & 4.89 & 1.53 & 0.14 & 10.9 & 14.7 & 8.3 & 56.5 & \\
\hline & V GCvBv & $69-92$ & $10 Y R 4 / 4$ & $s^{\prime} L$ & plat & $<10$ & 1 & Skelettlängsachsen senkr. & 4.84 & 1.01 & 0.09 & 11.2 & 11.1 & 4.8 & 43.2 & \\
\hline & V GB CV & $92-115$ & 10YR $4 / 4$ & $s^{\prime} L$ & plat & 10 & md & Skelettlängsachsen senkr. & 5.13 & 0.39 & 0.04 & 9.8 & 8.2 & 2.3 & 28.0 & \\
\hline & VIGCV & $115-170$ & 1OYR $5 / 2$ & $S$ & plat & 70 & $d$ & Kryoturbationen & 5.58 & 0.39 & 0.04 & 9.8 & 6.4 & 3.9 & 60.9 & \\
\hline & VII CV & $170-200+$ & $7.5 Y R 3 / 1$ & $\mathrm{~s}$ & $\sin$ & 80 & 1 & Schotter mit Verwitt.rind. & 5.25 & 0.15 & 0.03 & 5.0 & 8.8 & 2.4 & 27.2 & \\
\hline $\mathrm{K} 94 / \mathrm{T} 1 \mathrm{~b}$ & Te1 + Ah & 0.8 & $7.5 Y R 3 / 2$ & sU & fkrü & 0 & sl & & 4.37 & 13.18 & 0.89 & 14.8 & 4.8 & 2.9 & 60.4 & \\
\hline \multirow{9}{*}{ T1-Terrasse } & $\|$ Ah & $8-14$ & $7.5 Y R 3 / 4$ & $\mathrm{su}$ & fkrü & 0 & 1 & & 4.35 & 4.84 & 0.34 & 14.2 & 11.6 & 23.2 & 200.0 & \\
\hline & Te2 & $14-19$ & 10YR 4/6 & us & $\sin$ & 0 & 1 & & 4.54 & 2.57 & 0.10 & 25.7 & 8.1 & 6.9 & 85.1 & \\
\hline & III Ah & $19-28$ & 1OYR $3 / 4$ & $u$ & subpol-krü & 0 & 1 & & 4.68 & 5.13 & 0.38 & 13.5 & 7.9 & 17.5 & 221.5 & \\
\hline & Te3 & $28-43$ & 10YR $4 / 6$ & su & subpol & 0 & 1 & & 4.90 & 4.44 & 0.30 & 14.8 & 26.4 & 20.4 & 77.2 & \\
\hline & IV Ah & $43-46$ & 7.5YR $4 / 4$ & uL & subpol & 0 & md & & 4.87 & 4.54 & 0.31 & 14.6 & 26.5 & 15.1 & 56.9 & \\
\hline & IV GoBv & $46-56$ & 10YR $4 / 6$ & uL & subpol & 0 & md & & 4.67 & 4.48 & 0.34 & 13.2 & 24.2 & 20.2 & 83.4 & \\
\hline & IV BvGo & $56-60$ & $10 Y R 4 / 6$ & IU & subpol & 0 & md & & 4.67 & 1.81 & 0.14 & 12.9 & 13.5 & 9.1 & 67.4 & \\
\hline & V Go Ah & $60-65$ & $7.5 Y R 4 / 4$ & IU & plat & 0 & md & & 4.70 & 1.93 & 0.15 & 12.9 & 20.7 & 12.7 & 61.3 & \\
\hline & V Go & $65-82$ & 10YR $5 / 4$ & $\mathrm{uL}$ & plat & $<5$ & sd & & 4.71 & 0.57 & 0.05 & 11.4 & 10.2 & 5.7 & 55.8 & \\
\hline \multirow[t]{2}{*}{$\mathrm{K} 94 / \mathrm{T} 1 \mathrm{~b}$} & VI Go Cv & $82-102$ & 2.5 YR $5 / 3$ & $\mathrm{uL}$ & plat & $<10$ & sd & & 5.31 & 0.39 & 0.04 & 9.8 & 5.1 & - & & \\
\hline & $\mathrm{VIICV}$ & $102-125+$ & 2.5 YR $5 / 3$ & $\mathrm{~L}$ & plat & $40-50$ & $d$ & & 5.33 & 0.30 & 0.04 & 7.5 & 5.2 & - & & \\
\hline
\end{tabular}




\begin{tabular}{|c|c|c|c|c|c|c|c|c|c|c|c|c|c|c|c|}
\hline K 94/M1a & Ah & $0-7$ & 7.5YR $2 / 1$ & fsU & fkrü & 0 & sl & & 4.75 & 11.79 & 0.69 & 17.1 & 3.8 & 2.4 & 63.1 \\
\hline M1-Moräne & $A e=T e 1$ & $7-11$ & 7.5YR $6 / 2$ & $\mathrm{~s}$ & $\sin$ & 0 & sl & & 4.30 & 1.58 & 0.11 & 14.4 & 2.8 & 1.7 & 60.7 \\
\hline am Hang & $\| A h+T e 2$ & $11-25$ & $7.5 Y R 3 / 4$ & $\mathrm{sU}$ & subpol & 0 & 1 & & 4.46 & 2.94 & 0.19 & 15.5 & 12.5 & 10.7 & 85.6 \\
\hline \multirow[t]{5}{*}{ am Hang } & IIIGBAh & $25-45$ & $7.5 Y R 3 / 4$ & $\mathrm{sU}$ & subpol & 0 & 1 & & 4.62 & 5.71 & 0.37 & 15.4 & 20.2 & 16.2 & 80.1 \\
\hline & Te3 & $45-55$ & 5YR 4/6 & su & subpot & 0 & 1 & & 4.73 & 5.72 & 0.34 & 16.8 & 23.1 & 17.7 & 76.6 \\
\hline & IV Ah & $55-60$ & $7.5 Y R 4 / 4$ & stL & plat & 10 & $\mathrm{md}$ & Ton-Cutane a.Aggregatoberfl. & 4.64 & 7.93 & 0.45 & 17.6 & 37.1 & 27.8 & 74.9 \\
\hline & V GBv & $60-67$ & $7.5 Y R 4 / 6$ & & & & & & & & & & & & \\
\hline & v GCvBv & $67-100+$ & $10 Y R 4 / 6$ & usL & plat & 60 & sd & Wasserstau & 4.66 & 1.75 & 0.12 & 14.6 & 19.2 & 5.3 & 27.6 \\
\hline K 94/M1b & Ah & $0-7$ & $7.5 Y R 2 / 1$ & $\mathrm{fs} \mathrm{U}$ & fkrü & 0 & sl & & & & & & & & \\
\hline M1-Moräne & $\mathrm{Ae}=\mathrm{Te} 1$ & $7-11$ & $7.5 Y R 6 / 2$ & $\mathrm{~s}$ & $\sin$ & 0 & sl & & & & & & & & \\
\hline schwach konkave & $B s=T e 1$ & $11-19$ & 10YR $5 / 4$ & s & $\sin$ & 0 & si & & & & & & & & \\
\hline \multirow[t]{11}{*}{ Lage } & \| Ah & $19-23$ & 10YR $3 / 2$ & su & subpol & 0 & 10 & & & & & & & & \\
\hline & Te2 & 23-29 & 10YR $5 / 3$ & $\mathrm{~s}$ & $\sin$ & 0 & 1 & & & & & & & & \\
\hline & III Ah & $29-38$ & 10YR $4 / 2$ & $\mathrm{u}$ & subpol & 0 & 1 & & & & & & & & \\
\hline & Te3 & $38-55$ & 5YR 4/6 & su & subpol & 0 & 1 & & & & & & & & \\
\hline & IV Ah & $55-60$ & 10YR $5 / 6$ & $\mathrm{u}$ & subpol & 0 & 1 & & 5.17 & 3.11 & 0.20 & 15.6 & 17.9 & 11.5 & 64.2 \\
\hline & Te4 & $60-65$ & 10YR 4/6 & U & subpol & 0 & 1 & & 5.12 & 2.65 & 0.17 & 15.6 & 16.8 & 11.7 & 69.6 \\
\hline & VAh & $65-70$ & 10YR $4 / 4$ & 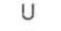 & subpo! & 0 & 1 & & 4.98 & 3.00 & 0.18 & 16.7 & 27.1 & 14.7 & 54.2 \\
\hline & V Go & $70-75$ & $7.5 Y R 4 / 6$ & $u$ & plat & $<10$ & 1 & rostfarben & 5.01 & 6.41 & 0.36 & 17.8 & 40.5 & 19.8 & 48.8 \\
\hline & VIG Bv & $75-90$ & 10YR 3/4 & $\mathrm{U}$ & plat & $<10$ & md & Skelettlängsachsen senkr. & 5.03 & 4.05 & 0.26 & 15.6 & 32.1 & 11.5 & 35.8 \\
\hline & VI GCV & $90-110$ & 1OYR $4 / 3$ & sL & plat & 20 & d & & 4.81 & 0.62 & 0.05 & 12.4 & 11.1 & 2.2 & 19.8 \\
\hline & VI GCn & $110-120+$ & 10YR $6 / 3$ & sL & plat & 50 & d & & 4.71 & 0.20 & 0.02 & 10.0 & 8.7 & 1.7 & 19.5 \\
\hline K 94/M2 & Ah & $0-8$ & $7.5 Y R 2 / 2$ & fsU & fkrü & 0 & sl & & 4.83 & 8.72 & 0.46 & 19.0 & 3.1 & 1.8 & 58.0 \\
\hline M2-Moräne & $A e=T e 1$ & $8-12$ & 7.5YR $5 / 3$ & $S$ & $\sin$ & 0 & 1 & & 4.55 & 1.01 & 0.07 & 14.4 & 1.9 & 1.2 & 63.1 \\
\hline schwach konkave & $B s=T e 1$ & $12-16$ & 10YR 5/6 & $\mathrm{s}$ & $\sin$ & 0 & 1 & & 4.58 & 1.42 & 0.09 & 15.8 & 4.2 & 4.1 & 97.6 \\
\hline \multirow[t]{11}{*}{ Lage } & II Ah & $16-25$ & 10YR $3 / 4$ & $\mathrm{sU}$ & subpol & 0 & 1 & & 4.56 & 2.88 & 0.17 & 16.9 & 8.3 & 8.2 & 98.7 \\
\hline & Te2 & $25-33$ & 7.5YR 4/6 & u'S & subpol & 0 & 1 & & 4.79 & 1.36 & 0.10 & 13.6 & 5.8 & 5.7 & 98.2 \\
\hline & III Ah & $33-40$ & $7.5 Y R 4 / 3$ & fsU & subpol & 0 & 1 & & 5.02 & 2.79 & 0.16 & 17.4 & 25.0 & 9.8 & 39.2 \\
\hline & Te3 & $40-60$ & $5 Y R 4 / 8$ & sU & subpol & 0 & 1 & & 5.42 & 2.81 & 0.19 & 14.8 & 24.4 & 15.2 & 62.2 \\
\hline & IV Ah & $60-65$ & 7.5 YR $5 / 6$ & U & subpol & 0 & 1 & & 5.48 & 3.01 & 0.20 & 15.1 & 27.9 & 16.2 & 58.0 \\
\hline & IV AhBv & $65-70$ & 7.5YR 4/6 & U & subpol & 0 & 1 & an der Basis dünne & 5.44 & 3.19 & 0.21 & 15.2 & 30.5 & 12.8 & 41.9 \\
\hline & V G Bv & $70-87$ & $7.5 Y R 4 / 4$ & $\mathrm{u}$ & subpol & $<10$ & 1 & Tephralagen (T4) & 5.18 & 2.49 & 0.19 & 13.1 & 23.0 & 13.8 & 60.0 \\
\hline & VI Go & $87-93$ & $7.5 Y R 4 / 4$ & $\mathrm{u}$ & subpol & $<10$ & $\mathrm{md}$ & & 5.22 & 3.79 & 0.28 & 13.5 & 65.3 & 40.7 & 62.3 \\
\hline & VII GAh & $93-97$ & 7.5YR 4/4 & $\cdot$ & $\cdot$ & $\cdot$ & $\cdot$ & & $\cdot$ & - & $\cdot$ & $\cdot$ & $\cdot$ & $\cdot$ & - \\
\hline & VIII GCv & $97-115$ & 7.5YR 4/4 & usL & plat & 70 & $\mathrm{md}$ & & 5.19 & 2.65 & 0.22 & 12.0 & 18.7 & 13.4 & 71.6 \\
\hline & VIII GCn & $115-140+$ & & sL & plat & 75 & md & & 5.31 & 1.28 & 0.11 & 11.6 & 23.6 & 13.4 & 56.7 \\
\hline
\end{tabular}

Fortsetzung Tab. 2 


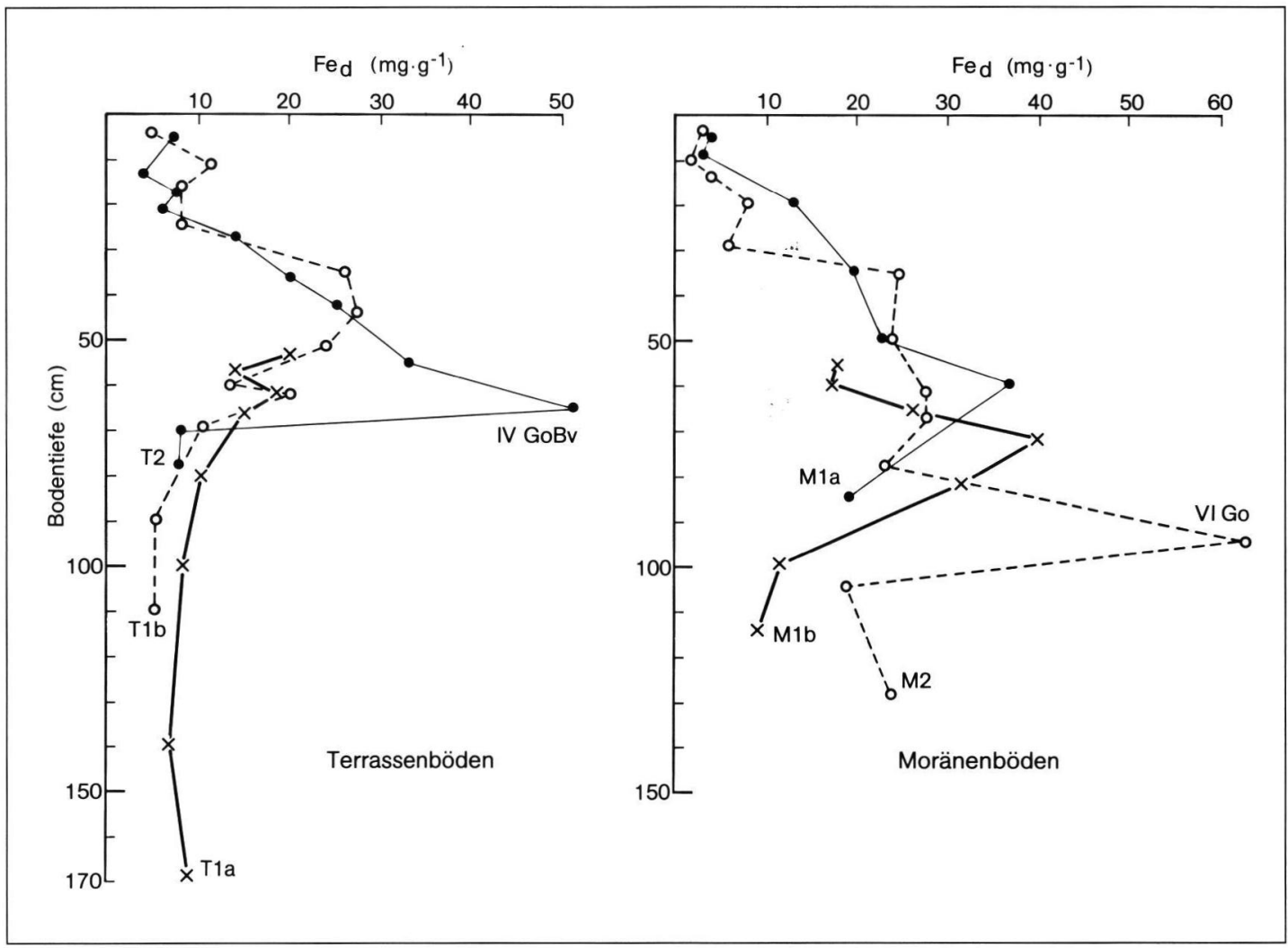

Abb. 5: Tiefenfunktion der $\mathrm{Fe}_{\mathrm{d}}$-Werte in Terrassenböden (links) und Moränenböden (rechts) des Plotnikova-Tales.

Fig. 5: Depth function of $\mathrm{Fe}_{\mathrm{d}}$-values in soils developed on terraces and drift of the Plotnikova Valley.

(z. T. mit Tephra 4 wie in Profil K94/B1, oder mit einem Go-Horizont wie in Profil K94/B2), die in verbraunte, rostfleckige, vielfach plattig-strukturierte GBv-Lagen übergehen.

Überblickt man diese Befunde, so gilt:

- Die Böden der M1- und M2-Moränen unterscheiden sich im Profilaufbau und in der Horizontfolge nicht wesentlich.

- Die Unterbodenverbraunung ist im Profil K94/M1a etwas deutlicher als in den Profilen K94/M1b und K94/M2. Die $\mathrm{Fe}_{\mathrm{O}} / \mathrm{Fe}_{\mathrm{d}}$-Quotienten schwanken unterhalb von Tephra 3 zwischen 0,27-0,75 in Profil M1a, zwischen $0,20-0,70$ in M1b und zwischen 0,42-0,72 in Profil M2. Die $\mathrm{Fe}_{\mathrm{d}}$-Werte lassen für den Unterboden nach Abb. 5 keine nennenswerten Unterschiede erkennen, wenn man wiederum von den durch Rostflecken bedingten Schwankungen absieht.

- Das Bodenskelett der M1-Böden scheint intensiver verwittert als jenes der M2-Böden. Vereinzelt haben die Steine der M1-Moränenböden geringmächtige Verwitterungsrinden.

Diese Ergebnisse bestätigen, daß die M1-Moränen ähnlich wie die T1-Terrassen im Vergleich zu den T2-Terrassen - zwar älter sind als die M2-Moränen, aber keine Anzeichen einer interglazialen, intensi- ven Verwitterung aufweisen. Im benachbarten Alaska sind mittelpleistozäne Ablagerungen stets wesentlich tiefgründiger verbraunt (bis zu mehreren Metern), als die spätpleistozänen Sedimente (PEwE 1975). Allerdings fehlen uns für definitive Aussagen absolute Datierungen von Moränen und Terrassen, sowie Analysendaten von eindeutig interglazialen Böden. Dringend erforderlich ist es, der Frage nach der maximalen Vergletscherung nachzugehen. Sie soll im Spätpleistozän erfolgt sein und bis an bzw. ins Meer gereicht haben. Ausgehend von unseren Befunden ist jedoch anzunehmen, daß die M1-Moränen während einer früheren Phase des Spätpleistozäns (vermutlich dem Frühwürm vergleichbar) abgelagert wurden, die M2-Moränen dagegen während einer späteren Periode (vermutlich vergleichbar mit dem Würmhochglazial). Die küstennahen Moränen wären dann mittelpleistozänen Alters, was allerdings noch genauer zu untersuchen ist. Sofern sich diese Vorstellungen bewahrheiten sollten, ergäbe sich ein deutlicher Unterschied zum Alpenraum, wo die Vergletscherung während der frühen Phasen des Spätpleistozäns weniger intensiv war als jene späterer Phasen. Dies entspricht auch den Vermutungen von VelichKo (1987), wonach die 
Vereisung im Osten Sibiriens im frühen Spätpleistozän aufgrund einer Verschiebung der Großwetterlagen und schnelleren Abkühlung über Sibirien wesentlich ausgeprägter war als in den nachfolgenden Phasen, während in Mitteleuropa wärmere atlantische Luftmassen die Abkühlung noch verzögerten. Im gleichen Zeitraum, als dann in Mitteleuropa aufgrund ausreichender Niederschläge die spätpleistozäne Maximalvergletscherung stattfand, baute sich in Sibirien und im ostasiatischen Raum eine ausgeprägte Hochdrucklage auf mit trockenem, kaltem Klima. Der ausgeprägte Unterschied in der Morphologie und den kryoturbaten Störungen zwischen M1und M2-Ablagerungen wäre damit gut erklärbar. Vergleichbare Ergebnisse zeigen auch Untersuchungen in den angrenzenden Gebieten Alaska (HAMILTON 1986) und Japan (YoshiKaWA et al. 1981).

Aufgrund fehlender absoluter Datierungen und den Befunden bezüglich der Tephrachronologie kann allerdings auch Hypothese E (M1 = Spätpleistozän, Phase 2; M2 = Spätglazial) nicht zweifelsfrei ausgeschlossen werden. Der relativ kurze Zeitraum reicht allerdings aus unserer Sicht nicht, um das Auftreten von deutlichen Verwitterungsrinden am Bodenskelett der M1- bzw. T1-Ablagerungen und die ausgeprägten kryoturbaten Störungen im Vergleich zu den M2- bzw. T2-Ablagerungen zu erklären. Unsere neu- esten Geländebefunde und Luftbildauswertungen von 1996 zeigen zudem, daß in einer Höhenlage zwischen 700-800 $\mathrm{m}$ ü. M. im Topolowaja- und im benachbarten Olkhowaja-Tal sowie in 1100-1300 m ü. M. im wesentlich trockeneren Teil der südlichen Zentral-Kamtschatka (Vaktan-Tal, Awatscha-Tal) eine weitere Generation von glazialen Ablagerungen vorhanden ist, die möglicherweise dem Spätglazial zugeordnet werden können. Eine Berechnung der Schneegrenze (ELA) oder deren Absenkung während vorzeitlicher Kältephasen könnte mehr Klarheit verschaffen, ist allerdings problematisch, da im Arbeitsgebiet keine rezente Vergletscherung mehr vorliegt und rezente wie vorzeitliche Hebungsraten für Kamtschatka nicht bekannt sind. Eine vorsichtige Kalkulation der Schneegrenzdepression für die M2-Ablagerungen ergibt unter der Annahme, daß die heutige Schneegrenze im Arbeitsgebiet bei etwa 1100-1200 m liegt, Werte um 400-450 $\mathrm{m}$. Dies würde nach Zugrundelegung der in Hochasien gefundenen Werte (KuHLe 1994) bedeuten, daß die 350-m-Moränen ins Spätglazial datiert werden könnten. Eine Erweiterung der Kalkulationen auf die anderen im Abschnitt 2.2 diskutierten glazialen Ablagerungen im Topolovaja-Tal (Abb. 6) zeigt, daß für die 980-m-Moränen die Schneegrenze um etwa $30 \mathrm{~m}$ abgesenkt war. Die dazugehörigen Glet-

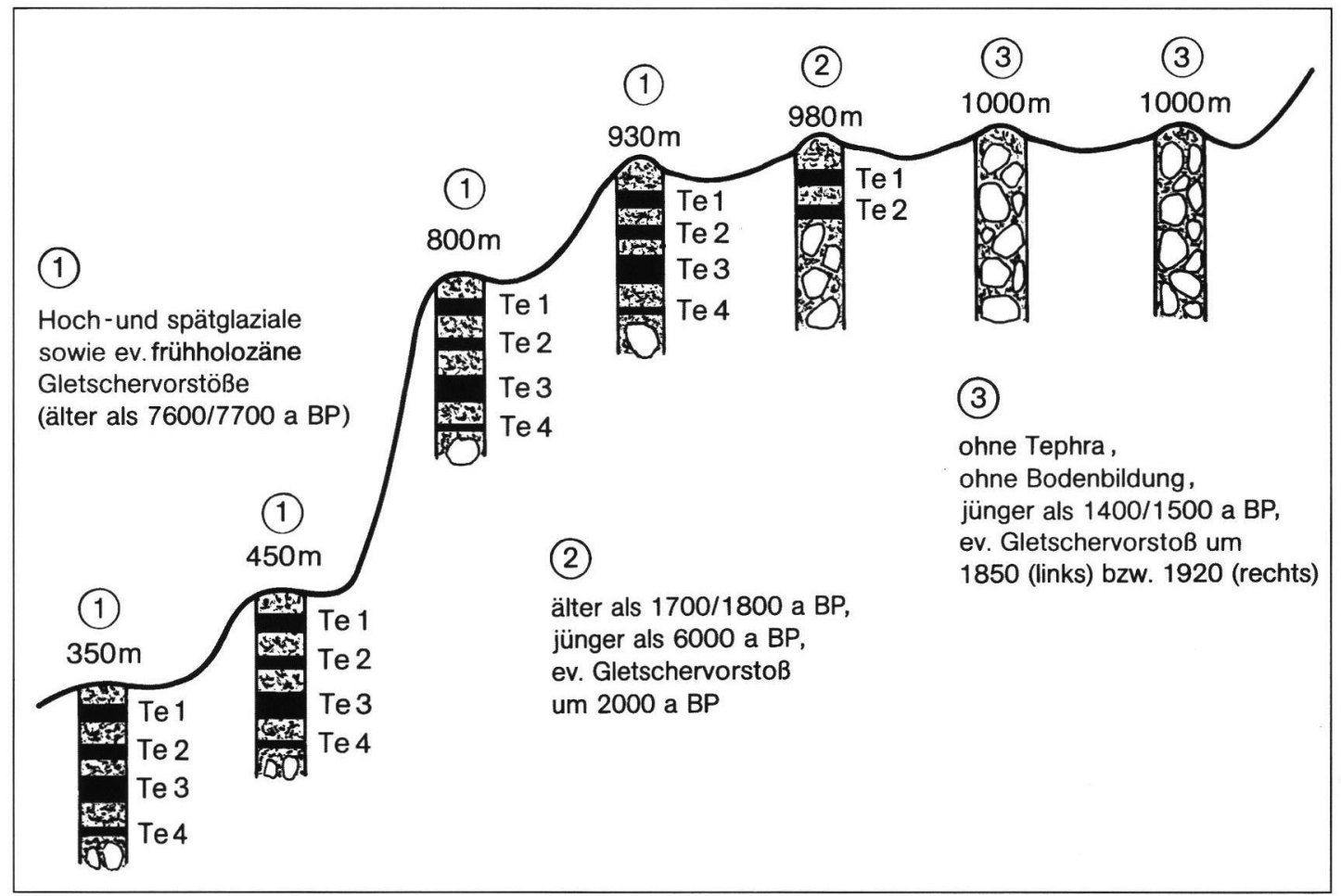

Abb. 6: Tephrastratigraphie von Böden im Topolovaya-Gebiet entlang eines Höhengradienten von 350 m (= M2-Moränen der Talvergletscherung) bis $1000 \mathrm{~m}$ ü. M. (= Moränen jungholozänen Alters).

Fig. 6: Tephra-stratigraphy of a soil catena from the M2-Valley drift in $350 \mathrm{~m}$ a.s.l. up to recent glacial deposits in $1000 \mathrm{~m}$ a.s.l., Topolovaja Valley. 
schervorstöße erfolgten aufgrund der Tephrastratigraphie in einem Zeitraum zwischen 6000 Jahre und 1700/1800 Jahre BP, d. h. im sog. Neoglazial. Und dies bedeutet wiederum eine um mindestens $70 \%$ unter den Vergleichswerten für Hochasien liegende Absenkung der ELA. Unter der weiteren Annahme, daß dieser Unterschied zwischen Hochasien und der Halbinsel Kamtschatka im gesamten Zeitraum ab dem Spätpleistozän bis heute Gültigkeit hat, würde eine Schneegrenzdepression von 400-450 m für die M2-Ablagerungen durchaus den in Hochasien gefundenen Werten von 1000-1200 m für Ablagerungen des Spätpleistozäns entsprechen.

Wenden wir uns im folgenden der zweiten Fragestellung zu.

\subsection{Profilaufbau der Böden mit zunehmender Höhenlage}

Im Abschnitt 2.1 wurde bereits die Tephrastratigraphie der Terrassen- und Moränenböden im Plotnikovatal vorgestellt. Aus der in Abb. 3 beschriebenen zeitlichen Zuordnung der Tephralagen folgt, daß das Alter eventueller holozäner Moränen aufgrund ihrer Tephralagen erfaßbar ist. Um dies zu prüfen, untersuchten wir den Profilaufbau der Böden von Stirnmoränenwällen entlang einer Höhensequenz im Topolovajatal von $350 \mathrm{~m}$ (= Stirnmoräne der M2-Talvergletscherung) bis auf etwa $1000 \mathrm{~m}$ ü. M. Abb. 6 informiert über die Ergebnisse. Man erkennt: - Die Moränen von 350 bis etwa 930 m ü. M. weisen die Tephralagen 1-4 auf; sie sind somit älter als die Kuril Lake Il'inskaya-Eruption (7600-7700 Jahre BP). Sie dokumentieren also spätpleistozäne bis frühholozäne Gletschervorstöße.

- Erst in $980 \mathrm{~m}$ ü. M. ändert sich die charakteristische Tephrastratigraphie. Es fehlen Tephralagen 3 und 4. Daraus folgt, daß die Moränen in etwa $980 \mathrm{~m}$ ü. M. älter als 1700/1800 Jahre BP sind, weil sie neben Tephra $1(=1400 / 1500$ Jahre BP $)$ nur noch Tephra 2 (1700/1800 Jahre BP) aufweisen; sie sind jedoch jünger als 6000 Jahre BP, da Tephra 3 (= 6000 Jahre BP) fehlt. Möglicherweise handelt es sich bei den 980-mMoränen um Ablagerungen mittelholozäner (neoglazialer) Gletschervorstöße.

- Die Moränen um etwa 1000 m ü. M. sind sehr jung und zeigen lediglich initiale Bodenbildung; Tephraablagerungen sind nicht zu erkennen. Neben der sehr geringen Verwitterungsintensität machen vergleichende lichenometrische Untersuchungen (SAvOSKUL et al. 1996) wahrscheinlich, daß diese höchstgelegenen Moränen mit Eisvorstößen während der sog. „kleinen Eiszeit" korrelieren, wie sie von Brartseva et al. (1968) für vergletscherte Vulkane in Kamtschatka nachgewiesen wurden.

Zusammenfassend gilt, daß die höhenzonale Untersuchung der Tephrastratigraphie von Moränenbö- den wertvolle Hinweise auf das Alter der Böden erlaubt. Methodisch gesehen ist es für weitergehende Untersuchungen angezeigt, in einem Gebiet mit rezenter Vergletscherung zu arbeiten und, von den Gletschern ausgehend, systematisch talabwärts den Profilaufbau der Böden zu überprüfen.

Weitere Untersuchungen auf der Basis glazialmorphologischer und bodengeographischer Methoden und unter Miteinbeziehung lichenometrischer und pollenanalytischer Befunde, sowie absoluter Altersdatierungen und Berechnungen von Schneegrenzdepressionen sind notwendig, um eine definitive Moränenstratigraphie Süd-Kamtschatkas zu erstellen.

\section{Dank}

Der Deutschen Forschungsgemeinschaft (436 RUS 113/6/0) und der Russischen Akademie der Wissenschaften danken wir verbindlichst für finanzielle Unterstützung. Für zahlreiche Hilfestellungen vor Ort sind wir besonders Frau Dr. BratTseva und Herrn Dr. Melekestsev (Institut für Vulkanische Geologie und Geochemie) sowie Herrn Dr. Muravjev (Institut für Vulkanologie) in Dankbarkeit verbunden.

\section{Schriftenverzeichnis}

Arkhipov, S. A.; Isayeva, L. L.; Bespaly, V. G.; Glushkova, O (1986): Glaciation of Siberia and north-east USSR. In Sibrava, V.; Bowen, D. Q. \& Richmond, G. M., Quarternary Glaciations in the Northern Hemisphere. Report of the Intern. Geological Correlation Programme, Project 24, Pergamon Press.

Bäumler, R.; ZeCh, W.; Heuberger, H.; Weber-Diefenbach, K. (1991): Investigations on the intensity of weathering of soils developed from glacial and fluvioglacial deposits and their relationship with the history of the landscape in the Mt. Everest region. Geoderma, 48: 223-243.

Braitseva, O. A.; Melekestsev, I. V.; Evteeva I. S.; Lupikina, Y. G. (1968): Stratigrafija tschetvjertitschnich otloschenij i oledenenija Kamschatki (Stratigraphy of Quaternary deposits and glaciations of Kamchatka), Moscow, Nauka, 226 S. (in Russian).

Melekestsev, I. V.; Ponomareva, V. V.; Kirianov, V. Yu.; LiTASOVA, S. N.; Sulerzhitsky, L. D. (1992): Tephra of the largest prehistoric Holocene volcanic eruption in Kamchatka. Quaternary International, 13/14: 177-180.

Hamilton, T. D. (1986): Late Cenozoic glaciation of the central Brooks Range. In: Hamilton, T. D.; ReED, K. M. Thorson, R. M. (eds.), Glaciation in Alaska - The geological record. Alaska geological society, 9-49.

Koschemianko N. N. (1966): Tschetvertitschnoe oledenenie Sredinnogo chrebta Kamschatki i voprosi rastschlenenija tschetvertitschnich effusivov (The quaternary glaciation of the Sredinnij range of Kamchatka and questions of age recognition of the Quaternary effusivs) In: Stratigraphija vulkanogennich formazij Kamtschatki. Moscow, Nauka. (in Russion).

KuHle, M. (1994): Present and Pleistocene glaciation on the north-western margin of Tibet between the Karakorum main ridge and the Tarim Basin, supporting the evidence of a Pleistocene inland glaciation in Tibet GeoJournal, 33.2/3: 133-272. 
Kuschev, S. L.; LiverovskiJ, J. A. (1938): Osnovnije momenti evoluzii phisikogeografitscheskich uslovij Zentralnoi Kamtschatskoi depressii $v$ tschetvertitschnom periode (The general features of the evolution of the physicogeographical conditions of the central Kamchatka depression during the Quaternary) Dokladi AN SSSR, novaja serija, t.19, vip.8: 67-81 (in Russian).

- (1940): Geomorphologitscheskij otscherk Zentralnoi Kamtschatskoi depressii (Geomorphological description of the central Kamchatka depression). - Trudi Instituta geographii AN SSSR, vip. 32, 87 S., Moscow (in Russian).

LAPSCHIN, L. I. (1963): O drevnem oledenenii Kamtschatki (About the older glaciation of Kamchatka). Voprosi geographii Kamtschatki, vip. 1: 62-64 (in Russian).

PEwe, T. L. (1975): Quaternary Geology of Alaska. Geological survey. Professional Paper 835, United States Government Printing Office, Washington.

SAvoskul, O. S.; SAuer, G.; ZeCH, W.; BÄumler, R. (1996): Holocene glacial fluctuations in the Topolovaya valley, S Kamchatka. Arctic and Alpine Research, submitted.

Solomina, O. N.; Muravjev, Y. D.; Basanova, L. I. (1995): Oledenenie Kamchatki $\mathrm{v}$ malom lednikovom periode (The Little Ice Age glaciation of Kamchatka). Izvestija Akademii Nauk SSSR, serija geographitscheskaja, in press (in Russian).

TscherbaKov, A. V. (1938): Dva geologitscheskich peresetschenija poluostrova Kamtschatki (Two geological profiles of the Kamchatka peninsula). Trudi SOPS AN SSSR, Serija Kamtschatskaja, vip. 5: 38-56, Moscow Leningrad (in Russian).

- (1940): Marschrutnije geologitscheskije issledovanija $v$ Sredinnom chrebtje (The route geological research in the Sredinnij range) Trudi SOPS AN SSSR, vip. 1: $58 \mathrm{~S}$., Moscow - Leningrad (in Russian).

- (1941): Geologitscheskije issledovanija po marschrutu s Kirganik (Geological research near Kirganik) S. Chalaktirka. - Trudi Kamtsch. kompl. excp. AN SSSR, vip. 3: $72 \mathrm{~S}$., Moscow - Leningrad (in Russian).

VelichKo, A. A. (1987): Present-day state of development of the concept of ice sheets. Izvestiya AN SSSR, ser. geogr. 3: 21-34 (in Russian).

Vlasov, G. M., Tschemenkov, J. F. (1950): Osnovije etapi formirovanija reljefa poluostrova Kamtschatki i ego geomorphologtscheskoe raijonirovanie (The most important stages of the relief formation of Kamchatka and its geomorphological mapping) - Isvestija Vsesojusnogo geographitscheskogo obtshestva, 1950, t.82, vip. 3: 262-272, (in Russian).

- (1959a): Visokije poverchnosti viravnivanija Kamtschatki i Kurilskich ostrovov (Uplifted peneplains of Kamchatka and the Kuril Islands) - Materiali geomorphologitscheskogo soveschanjia, vip. 2: 178-192, (in Russian).

- (1959 b): Osnovnije tscherti reljefa Kamtschatki i Kurilskich ostrovov (The general view on the relief of Kamchatka and Kuril Islands) Materiali II geomorphologitscheskogo soveschanija, vip. 1: 43-45, (in Russian).

Yoshikawa, T.; KaizuKa, S.; OTA, Y. (1981): The landforms of Japan. University of Tokyo Press, Tokyo, 202 S.

Manuskript eingegangen am 25.10.1995 